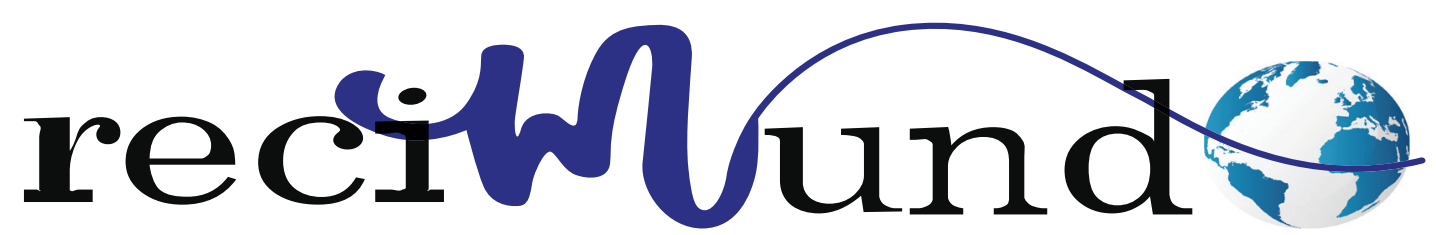

Revista Científica Mundo de la Investigación y el Conocimiento

DOl: 10.26820/recimundo/4.(4).octubre.2020.46-58

URL: http://recimundo.com/index.php/es/article/view/880

EDITORIAL: Saberes del Conocimiento

REVISTA: RECIMUNDO

ISSN: 2588-073X

TIPO DE INVESTIGACIÓN: Artículo de Revisión

CÓDIGO UNESCO: 3205 Medicina Interna

PAGINAS: $46-58$

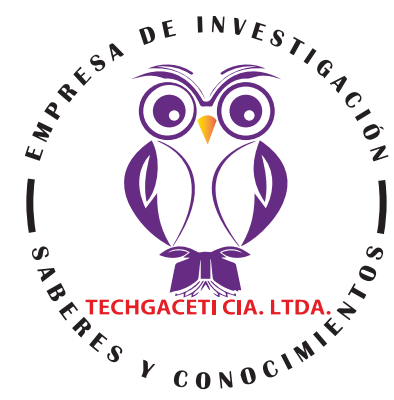

\title{
Manejo de hidratación en pancreatitis aguda
}

Hydration management in acute pancreatitis

Manejo da hidratação na pancreatite aguda

Isaac Fernando Díaz Castro1; Castillo Olvera Jonathan Alberto2; Susan Michelle Erazo Haro3;

María Fernanda Matute Solis ${ }^{4}$

RECIBIDO: 10/07/2020 ACEPTADO: 26/08/2020 PUBLICADO: 15/10/2020

1. Médico; Hospital Naval General; Guayaquil, Ecuador; isaacfernandodiazcastro@gmail.com; (iD https://orcid.org/00000003-2727-2105

2. Médico; Área de Emergencias; Hospital Naval de Guayaquil; Guayaquil, Ecuador; jonathan_athlerm@hotmail.com; https://orcid.org/0000-0002-1089-9297

3. Médico; Hospital Naval General; Guayaquil, Ecuador; suerazo18@gmail.com; (iD https://orcid.org/0000-0002-9075-0029

4. Médico; Hospital Naval General; Guayaquil, Ecuador; mafermatute14@gmail.com; (D) https://orcid.org/0000-0002-74887797

\section{CORRESPONDENCIA}

Isaac Fernando Díaz Castro

isaacfernandodiazcastro@gmail.com

Guayaquil, Ecuador

() RECIMUNDO; Editorial Saberes del Conocimiento, 2020 


\section{RESUMEN}

La pancreatitis aguda sigue siendo un desafío clínico, a pesar de un aumento exponencial del conocimiento de sus complejos cambios fisiopatológicos. La hidratación temprana es la piedra angular del tratamiento y se recomienda universalmente, sobre todo en las primeras horas del padecimiento de la enfermedad se considera realizar una hidratación agresiva, sin embargo, la sobrecarga de liquido puede generar riesgos en los pacientes, debido a que existe una falta de consenso con respecto al tipo, la velocidad, la cantidad y los puntos finales del reemplazo de líquidos. Por tal motivo el manejo de la hidratación debe ser controlada con el fin de brindar mejores resultados. Esta revisión se centra en la fisiopatología de la depleción de líquidos en la pancreatitis aguda, así como en la justificación de la reposición de líquidos, el tipo, la cantidad óptima, la velocidad de infusión y el seguimiento de dichos pacientes. El objetivo básico de la depleción de líquidos debe ser prevenir o minimizar la respuesta sistémica a los marcadores inflamatorios. Para esta revisión, se evaluaron críticamente varios estudios y revisiones que tienen relación con el tema y que fueron de gran aporte para la investigación.

Palabras clave: Pancreatitis aguda; Resucitación fluida; Terapia de fluidos agresiva; Cristaloides; Coloides.

\section{ABSTRACT}

Acute pancreatitis remains a clinical challenge, despite an exponential increase in understanding of its complex pathophysiological changes. Early hydration is the cornerstone of treatment and is universally recommended, especially in the first hours of the disease, aggressive hydration is considered, however, fluid overload can generate risks in patients, because there is a lack of consensus regarding the type, speed, amount and end points of fluid replacement. For this reason, the management of hydration must be controlled in order to provide better results. This review focuses on the pathophysiology of fluid depletion in acute pancreatitis, as well as the justification for fluid replacement, type, optimal amount, infusion rate, and follow-up of such patients. The basic goal of fluid depletion should be to prevent or minimize the systemic response to inflammatory markers. For this review, several studies and reviews that are related to the topic and that were of great contribution to the research were critically appraised.

Keywords: Acute pancreatitis; Fluid resuscitation; Aggressive fluid therapy; Crystalloids; Colloids.

\section{RESUMO}

A pancreatite aguda continua sendo um desafio clínico, apesar do aumento exponencial na compreensão de suas complexas alterações fisiopatológicas. A hidratação precoce é a pedra angular do tratamento e é universalmente recomendada, principalmente nas primeiras horas da doença, considera-se a hidratação agressiva, porém, a sobrecarga hídrica pode gerar riscos nos pacientes, pois não há consenso quanto ao tipo, velocidade, quantidade e pontos finais da reposição de fluidos. Por esse motivo, o gerenciamento da hidratação deve ser controlado para proporcionar melhores resultados. Esta revisão enfoca a fisiopatologia da depleção de fluidos na pancreatite aguda, bem como a justificativa para a reposição de fluidos, tipo, quantidade ideal, taxa de infusão e acompanhamento de tais pacientes. O objetivo básico da depleção de fluidos deve ser prevenir ou minimizar a resposta sistêmica aos marcadores inflamatórios. Para esta revisão, diversos estudos e revisões que se relacionam com o tema e que foram de grande contribuição para a pesquisa foram avaliados criticamente.

Palavras-chave: Pancreatite aguda; Reanimação com fluidos; Terapia de fluidos agressiva; Cristaloides; Colóides. 


\section{Introducción}

La pancreatitis aguda (PA) puede manifestarse como una forma grave, que tiene una alta tasa de mortalidad. El tratamiento de la PA es principalmente de apoyo y la terapia de reemplazo de líquidos se ha convertido en una de las estrategias de tratamiento clave. Hay una falta de estudios aleatorios que aborden las preguntas del mejor tipo de líquido, la cantidad de líquido y la tasa de transfusión de líquido.

La pancreatitis aguda (PA) "es una inflamación del páncreas y tiene altas tasas de morbilidad y mortalidad" (Lowenfels, Maisonneuve, \& Sullivan, 2009). La PA muestra un amplio espectro de presentación de la enfermedad, que va desde una enfermedad leve autolimitada hasta una enfermedad grave rápidamente progresiva que termina en insuficiencia multiorgánica con un alto riesgo de mortalidad. "Se han descrito diferentes etapas de gravedad en la clasificación de Atlanta, se estima que alrededor del 10 al 20\% de los pacientes con PA desarrollan la forma grave, que tiene una tasa de mortalidad del 15 al 40\%" (Banks, y otros, Classification of acute pancreatitis--2012: revision of the Atlanta classification and definitions by international consensus, 2013).

Un factor importante que complica el manejo adecuado de la PA es la falta de discriminación de sus formas leve y grave en las etapas iniciales. Este problema es crítico, ya que "aproximadamente la mitad de los pacientes con PA grave mueren durante la primera semana debido al desarrollo de insuficiencia orgánica;la incidencia de insuficiencia orgánica es máxima (17\%) el primer día" (Wu \& Conwell, 2010). Las causas de la mortalidad posterior son el desarrollo de necrosis infectada y otras complicaciones. Por lo tanto, "es importante identificar los factores que pueden predecir la gravedad de la enfermedad de AP para orientar el manejo clínico temprano dentro de la denominada ventana intervencionista" (Tenner, 2014).
A pesar de una mayor comprensión de la fisiopatología subyacente tanto de la enfermedad como de sus complicaciones, su manejo sigue siendo un desafío clínico y se basa principalmente en la terapia de apoyo. La reanimación con líquidos es la piedra angular actual del manejo temprano, aunque hay poco consenso sobre los detalles de su aplicación. Las guías de práctica clínica ampliamente aceptadas recomiendan la reanimación vigorosa con líquidos en el tratamiento temprano de la PA. Sin embargo, existe una falta de consenso sobre recomendaciones específicas con respecto al tipo de líquido, la velocidad óptima de administración de líquidos y los puntos finales para indicar una reanimación adecuada.

Aunque para Mao, Tang, Li, \& Qin, (2017) "la hidratación agresiva se considera la terapia de primera en el tratamiento de la PA, algunos estudios recientes han sugerido que la hidratación no agresiva puede ser mejor para reducir la mortalidad y mejorar los resultados". Una revisión sistemática reciente ha puesto de relieve la falta de pruebas en la literatura sobre el tipo de líquido, la velocidad de infusión de líquido y los objetivos que deben controlarse. Incluso la evidencia disponible es de baja o muy baja calidad. Por lo tanto, se revisará la literatura sobre la reanimación con líquidos en el curso de la PA, haciendo énfasis en los objetivos, la elección y la cantidad de líquidos para reducir complicaciones como la necrosis pancreática y la falla orgánica.

\section{Metodología}

Para el desarrollo de este proceso investigativo, se plantea como metodología la encaminada hacia una orientación científica particular que se encuentra determinada por la necesidad de indagar en forma precisa y coherente una situación, en tal sentido Davila, (2015) define la metodología "como aquellos pasos anteriores que son seleccionados por el investigador para lograr resul- 
tados favorables que le ayuden a plantear nuevas ideas" (p.66)

Lo citado por el autor, lleva a entender que el desarrollo de la acción investigativa busca simplemente coordinar acciones enmarcadas en una revisión bibliográfica con el fin de complementar ideas previas relacionadas Manejo de hidratación en pancreatitis aguda a través de una revisión de literatura, para así finalmente elaborar un cuerpo de consideraciones generales que ayuden a ampliar el interés propuesto.

\section{Tipo de Investigación}

Dentro de toda práctica investigativa, se precisan acciones de carácter metodológico mediante las cuales se logra conocer y proyectar los eventos posibles que la determinan. En este sentido, la presente investigación corresponde al tipo documental, definido por Castro (2016), "se ocupa del estudio de problemas planteados a nivel teórico, la información requerida para abordarlos se encuentra básicamente en materiales impresos, audiovisuales y / o electrónicos". (p.41).

En consideración a esta definición, la orientación metodológica incluye la oportunidad de cumplir con una serie de actividades inherentes a la revisión y lectura de diversos documentos, donde se encuentran ideas explicitas relacionadas con los tópicos encargados de identificar una característica inmersa en el estudio. Por lo tanto, se realizaron continuas interpretaciones con el claro propósito de revisar aquellas apreciaciones propuestas por diferentes investigadores en relación al tema de interés, para luego dar la respectiva argumentación a los planteamientos, en función a las necesidades encontradas en la investigación, apoyados en las herramientas tecnológicas para la búsqueda de trabajos con valor científico disponibles en la web que tenían conexión con el objetivo principal de la investigación.

\section{Fuentes Documentales}

El análisis correspondiente a las características que predomina en el tema seleccionado, llevan a incluir diferentes fuentes documentales encargadas de darle el respectivo valor científico y en ese sentido cumplir con la valoración de los hechos a fin de generar nuevos criterios que sirven de referencia a otros procesos investigativos. Para Castro,(2016) las fuentes documentales incorporadas en la investigación documental o bibliográfica, "representa la suma de materiales sistemáticos que son revisados en forma rigurosa y profunda para llegar a un análisis del fenómeno" (p.41). Por lo tanto, se procedió a cumplir con la lectura previa determinada para encontrar aquellos aspectos estrechamente vinculados con el tema, con el fin de explicar mediante un desarrollo las respectivas apreciaciones generales de importancia.

\section{Técnicas para la Recolección de la Infor- mación}

La conducción de la investigación para ser realizada en función a las particularidades que determinan a los estudios documentales, tiene como fin el desarrollo de un conjunto de acciones encargadas de llevar a la selección de técnicas estrechamente vinculadas con las características del estudio. Bolívar, (2015), refiere, que es "una técnica particular para aportar ayuda a los procedimientos de selección de las ideas primarias y secundarias". (p.71).

Tal como lo expresa, Bolívar, (2015) "Las técnicas documentales proporcionan las herramientas esenciales y determinantes para responder a los objetivos formulados y llegar a resultados efectivos" (p. 58). Es decir, para responder con eficiencia a las necesidades investigativas, se introdujeron como técnica de recolección el método inductivo, que hizo posible llevar a cabo una valoración de los hechos de forma particular para llegar a la explicación desde una vi-

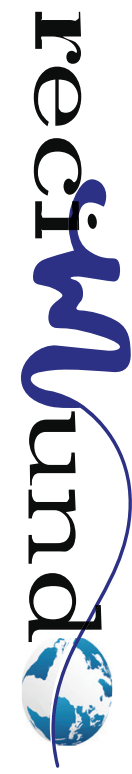


sión general. El autor Bolívar, (2015) tambien expresa que las técnicas de procesamiento de datos en los estudios documentales "son las encargadas de ofrecer al investigador la visión o pasos que deben cumplir durante su ejercicio, cada una de ellas debe estar en correspondencia con el nivel a emplear" (p. 123). Esto indica, que para llevar a cabo el procesamiento de los datos obtenidos una vez aplicadas las técnicas seleccionadas, tales como: fichas de resumen, textual, registros descriptivos entre otros, los mismos se deben ajustar al nivel que ha sido seleccionado.

\section{Resultados}

\section{Base patofisiológica}

Los principales factores etiológicos de la PA son el alcohol y la obstrucción biliar; otras causas son autoinmunidad, drogas, trauma, iatrogénica e idiopática. Los autores Mayer, Rau, Gansauge, \& Beger, (2010) expresan que "una complicación importante de la enfermedad es la insuficiencia orgánica como resultado de la activación excesiva de una cascada de respuesta inflamatoria sistémica". El daño pancreático debido a cualquier etiología conduce a la "liberación de mediadores proinflamatorios, como zimógenos, citocinas y factores vasoactivos. Estos mediadores provocan la activación de las células endoteliales que conducen a vasoconstricción arteriolar, aumento de la permeabilidad y estasis circulatoria, lo que induce isquemia" (Mayer, Rau, Gansauge, \& Beger, 2010).

Este aumento de la permeabilidad, relacionado con la fuga capilar, provoca pérdida de líquido intravascular e hipotensión, y puede sobrevenir un shock (Figura 1). Aunado a esto "la acumulación de mediadores inflamatorios con leucocitos mejorados y la interacción endotelial da como resultado activación de la cascada de coagulación e hipercoagulación. La trombosis microvascular provoca hipoxia tisular y, en última ins- tancia, síndrome de respuesta inflamatoria sistémica (SRIS)" (Hack \& Zeerleder, 2011). La disfunción orgánica suele ocurrir bastante temprano en el curso de la PA grave, por lo general "los primeros cuatro días, a menos que se realice un tratamiento agresivo, causa mortalidad en aproximadamente el $50 \%$ de los casos dentro de la primera semana de su manifestación" (Hack \& Zeerleder, 2011). Los primeros cinco días después del inicio de la enfermedad aguda, se consideran la "ventana de intervención terapéutica", durante la cual la reanimación intensiva con líquidos puede corregir las pérdidas del tercer espacio y aumentar la perfusión tisular. EI SRIS puede evitarse con la prevención de la insuficiencia orgánica múltiple y / o la necrosis pancreática.

\section{Clasificación de la severidad de la pan- creatitis aguda}

La gravedad de la PA puede clasificarse en leve (sin complicaciones locales o sistémicas), moderada (en la que las complicaciones locales o sistémicas se asocian con insuficiencia orgánica que se resuelve en 48 h) o grave (en la que la insuficiencia orgánica persiste más allá de las 48 h). "El riesgo de mortalidad es nulo o mínimo en pacientes con insuficiencia orgánica mínima o nula, y puede llegar al $15 \%$ al $40 \%$ en aquellos con enfermedad grave" (Banks \& Freeman, 2016). La insuficiencia orgánica se ha convertido en un determinante clave de la gravedad de la PA clasificándose como transitoria o persistente según la duración y de acuerdo a la puntuación de Marshall modificada.

Hay varios criterios de puntuación descritos en la literatura para evaluar la gravedad de la pancreatitis. Sin embargo, estos sistemas de puntuación requieren 48 h para completarse. Los autores (Johannes, Sun, \& Tabak, 2008) expresan que "las primeras 12-24 h son críticas, ya que, la máxima incidencia de desarrollo de insuficiencia orgánica se ajusta a este período de tiempo". Por tanto, 
recientemente se han propuesto dos nuevos sistemas de puntuación, cada uno de los cuales evalúa la gravedad en las primeras $24 \mathrm{~h}$.

El índice de gravedad de cabecera en pancreatitis aguda (BISAP) es uno y evalúa 5 criterios:

Nitrógeno ureico en sangre $(B U N)>25$ mg / dl, edad> 60 años, deterioro del estado mental, SRIS y derrame pleural. Para BISAP, una puntuación de $>2$ se asocia con un aumento de 10 veces en el riesgo de mortalidad. El segundo sistema de puntuación es la puntuación de pancreatitis aguda inofensiva, que se centra en pacientes que tienen pocas probabilidades de desarrollar pancreatitis grave. (Johannes, Sun, \& Tabak, 2008).

\section{Fundamentos de la reanimación de flui- dos}

El manejo de la PA gira en torno a la atención de apoyo, la nutrición adecuada y la hidratación intravenosa. La justificación de la hidratación se basa en la necesidad de resolver la hipovolemia que se presenta secundaria a vómitos, disminución de la ingesta oral, extravasación del tercer espacio, pérdidas respiratorias y diaforesis. Además, la hidratación temprana proporciona apoyo macro circulatorio y micro circulatorio para prevenir la cascada de eventos que conducen a la necrosis pancreática.

Se ha documentado que la "corrección de la hipovolemia, evaluada por los cambios en el hematocrito, el BUN y la creatinina sérica, limita la necrosis y mejora el resultado" (Wu \& Conwell, 2010). La hemoconcentración, como marcador de hipovolemia y gravedad de la pancreatitis, se ha estudiado desde la década de 1960.

Se informó que un hematocrito $\geq 44 \%-47 \%$ en el momento del ingreso combinado con el fracaso de una disminución del hemato- crito a las $24 \mathrm{~h}$ era el mejor factor de riesgo para el desarrollo de necrosis demostraron que la hemoconcentración temprana se asoció con un aumento de la mortalidad solo entre los casos transferidos al hospital y no entre los casos no transferidos. (Wu \& Conwell, 2010)

Esta diferencia podría deberse a variaciones en el manejo temprano de los casos estudiados, enfatizando aún más el hecho de que la reanimación con líquidos debe ser instituida de manera temprana. Similar, los cambios en BUN y creatinina desde el inicio son indicativos de depleción del volumen intravascular y ambos marcadores se utilizan para predecir el resultado. "El seguimiento de estos parámetros puede medir la eficacia de las medidas iniciales de reanimación. Por lo tanto, estos parámetros se pueden utilizar para optimizar la reanimación dirigida por objetivos" (Wu \& Conwell, 2010).

Las alteraciones micro circulatorias en la PA son diferentes de la simple hipovolemia por traumatismo o hemorragia, ya que son causadas por SIRS con sobreexpresión de mediadores inflamatorios que lesionan el endotelio y aumentan la permeabilidad capilar, lo que lleva a secuestro de líquidos y síndrome de extravasación capilar. Por tanto, el propósito de la reanimación con líquidos eficaz en la PA grave no es sólo reponer el volumen sanguíneo sino también estabilizar la permeabilidad capilar, modular la reacción inflamatoria y mantener la función de barrera intestinal.

\section{¿Qué pacientes requieren reanimación con líquidos?}

El objetivo principal de la hidratación en la pancreatitis aguda es limitar o prevenir la necrosis pancreática. Cualquier paciente con PA tiene el potencial de progresar a una enfermedad grave. Los pacientes con pancreatitis intersticial leve suelen permanecer en observación en la sala de emergencias

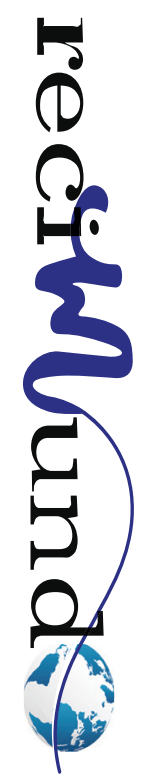


y, una vez que el dolor se calma, pueden ser dados de alta. Sin embargo, los pacientes con comorbilidades subyacentes requerirían una observación más cercana. Las directrices revisadas de Atlanta permiten clasificar y evaluar la gravedad de los pacientes. Los pacientes con PA moderada y grave requieren observación por insuficiencia orgánica y complicaciones locales o sistémicas, y deben comenzar con la fluidoterapia. Debe reconocerse que en el momento de la primera interfaz con el paciente puede no ser posible medir la gravedad, que puede evolucionar en las próximas 24-48 h.

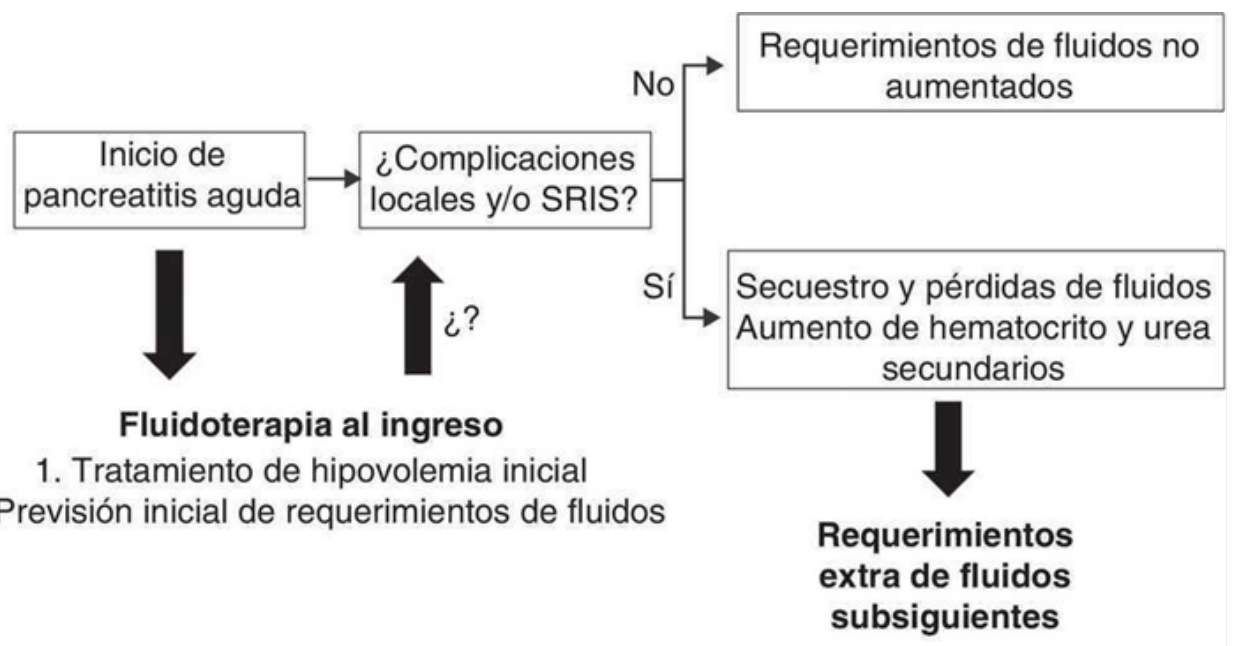

SIRS: síndrome de respuesta inflamatoria sistémica

Figura 1. Fisiopatología de la depleción de líquidos en pancreatitis.

Fuente: (Banks \& Freeman, 2016)

\section{Elección de fluido}

Dos tipos de líquidos que se utilizan con frecuencia son los coloides y los cristaloides. Los coloides comúnmente utilizados son diversas formulaciones de dextrano, hetastarch y albúmina. "Los coloides se consideran superiores a los cristaloides en la optimización de la respuesta hemodinámica, también tienen una mejor retención en el compartimento intravascular debido a su mayor tamaño" (Javier \& Ángel, 2015). Como permanecen dentro de la luz, a pesar del aumento de la permeabilidad vascular, ayudan a mantener un mejor flujo circulatorio. Además, contribuyen a la corrección de la hipovolemia debido a su efecto osmótico en la extracción de líquido del intersticio al compartimento vascular. Sin embargo, los coloides pueden causar sobrecarga de volumen intravascular, insuficiencia renal hipe- roncótica, coagulopatía y reacción anafiláctica.

Los cristaloides de uso común son la solución salina normal (NS), el lactato de Ringer $(R L)$ y el piruvato de etilo de Ringer, siendo la solución salina hipertónica el llamado "niño nuevo en el bloque". De acuerdo con Sterns, Riggs, \& Schochet, (2006) "Ios cristaloides se distribuyen tanto en el plasma como en los compartimentos intersticiales, por lo que se requieren grandes espacios para restaurar la circulación, sin embargo, la infusión de grandes cantidades de cristaloides podría provocar edema pulmonar". La solución salina hipertónica reduce eficazmente el volumen de reanimación con líquido isotónico, lo que reduce el riesgo de edema pulmonar.

Sin embargo, existe un riesgo potencial de 
mielinólisis pontina central con el tratamiento agresivo con solución salina hipertónica Aún no se ha determinado el líquido ideal para la reanimación en PA. La elección es principalmente entre un coloide y un cristaloide. La variabilidad en los resultados de los estudios iniciales con reanimación con fluidos podría atribuirse a los diferentes tipos de fluidos utilizados. Se ha demostrado que los coloides son superiores a los cristaloides en experimentos con animales, lo que puede deberse al hecho de que no son tan permeables a las fugas en la microcirculación pancreática como los cristaloides. Al permanecer en la luz, el flujo sanguíneo circulatorio se mantiene mejor y es menos probable que los mediadores inflamatorios alcancen el acino cuando se utilizan coloides. (Gardner, Vege, Pearson, \& Chari, 2018).

La mayoría de los estudios en animales sobre reanimación con fluidos han utilizado soluciones coloides y han descubierto que son mejores que los cristaloides

El uso de coloides en humanos ha involucrado varias preparaciones de dextrano y albúmina. En un estudio de "fase I en humanos en el que participaron 13 pacientes con pancreatitis no biliar grave, después de la hemodilución con dextrano, la progresión de necrosis pancreática se observó en el $15 \%$ y la mortalidad se observó en sólo el 7,7\%" (Foitzik, Buhr, Messmer, \& Herfarth, 2003).

El hidroxietil almidón (HES) es otro líquido coloide que puede preservar la oxigenación sistémica en pacientes con fuga capilar. "Si bien se ha demostrado que reduce el riesgo de hipertensión intraabdominal en la PA grave, no se ha observado ningún impacto sobre la insuficiencia orgánica y la mortalidad" (Zhao, Zhang, \& Wu, 2015). En un estudio reciente que comparó NS (grupo 1), HES y NS (grupo 2) y HES, NS y glutamina (grupo 3), Zhao, Zhang, \& Wu, (2015) demostraron que "los pacientes de los grupos
2 y 3 tenían tasas reducidas de insuficiencia orgánica y infección". Otras soluciones utilizadas en la reanimación con fluidos son "el transportador de oxígeno basado en hemoglobina libre de células, una combinación de hemodilución isovolémica con HES y un transportador de oxígeno plasmático, almidón de hidroxietilo al 6\% y furosemida" (Javier \& Ángel, 2015).

En resumen, existe una falta de evidencia de alto nivel para orientar la elección del líquido en PA. Los cristaloides son recomendados por la Asociación Americana de Gastroenterología, y "los coloides (concentrado de hematíes) se consideran en casos de hematocrito bajo (<25\%) y albúmina sérica baja $(<2 \mathrm{~g} / \mathrm{dl})$. Entre los cristaloides, se prefiere la solución RL a la NS" (Zhao, Zhang, \& Wu, 2015). Sin embargo, existe una necesidad urgente de estudios sobre este tema.

\section{Concepto de hidratación controlada}

Dado que se ha informado de un efecto secundario negativo para la reanimación intensiva con líquidos, algunos médicos han propuesto una reanimación con líquidos más controlada en lugar de una terapia con líquidos excesivamente entusiasta. "Un estudio chino de 83 pacientes confirmó las observaciones de que las tasas de supervivencia mejoraron significativamente al controlar la reanimación con líquidos y prevenir el secuestro de líquidos corporales" (Mao, Tang, Li, \& Qin, 2017).

Se ha señalado que hay un número igual de estudios a favor de la reanimación con líquidos agresiva y no agresiva.

Se puede argumentar que la reanimación agresiva restaura el compartimento intravascular agotado por el "tercer espaciamiento y da como resultado una perfusión tisular del órgano terminal más eficaz e invierte la isquemia pancreática. Los partidarios de la hidratación no agresiva sugieren

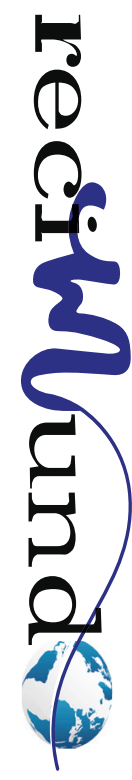


que cuando se interviene en pacientes con PA, la necrosis pancreática ya es irreversible y la fluidoterapia agresiva sólo conducirá a insuficiencia respiratoria y aumento de la presión intraabdominal, etc. Por lo tanto, una reanimación "controlada" destinada a revertir la hipotensión y poder mantener la presión arterial media efectiva (PAM) y la producción de orina> 0,5 ml / kg, es la mejor opción (Sarr, 2013).

\section{¿Cuánto líquido se secuestra?}

Las recomendaciones de diversas sociedades y grupos han sugerido una reanimación intensiva con líquidos en la PA; sin embargo, estas recomendaciones no se basaron en datos concretos. Aunque es bien sabido que algunos pacientes con PA tienen una mayor necesidad de terapia con líquidos, no está claro quién debe recibir líquidos de manera agresiva.

\section{Situaciones especiales}

Los pacientes con comorbilidades como insuficiencia renal, compromiso cardíaco y enfermedad pulmonar necesitan atención especial. "Según el sistema de clasificación revisado de Atlanta, las comorbilidades son determinantes e importantes de la gravedad de la PA" (Banks, Bollen, Dervenis, \& Gooszen, 2013). Además, la aparición de insuficiencia orgánica como consecuencia de la PA requiere una atención especial en estos pacientes. El tratamiento de estos pacientes puede extrapolarse de las directrices de la campaña Surviving Sepsis Campaign para el tratamiento del shock séptico. Para Dellinger, Carlet, \& Masur, (2004) los problemas en estos pacientes que necesitan consideraciones especiales "incluyen la tasa de líquido transfundido, el uso de agentes adicionales como vasopresores y la necesidad de un control específico". Puede ser necesario restringir la infusión de líquidos en aquellos pacientes que tienen disfunción renal o disfunción cardíaca". Como en los pacientes con sepsis, se re- quiere terapia vasopresora para mantener la presión de perfusión en caso de hipotensión, incluso cuando la hipovolemia aún no ha sido tratada. "Algunos pacientes requieren vasopresores para lograr una presión de perfusión mínima. En pacientes con sepsis, se ha demostrado que la titulación de la noradrenalina a una PAM tan baja como 65 mmHg conserva la perfusión tisular" (Dellinger, Carlet, \& Masur, 2004).

La noradrenalina es el vasopresor recomendado en tales situaciones. El tratamiento del síndrome de dificultad respiratoria del adulto se realiza según las directrices estándar.

Recientemente, se ha demostrado que el soporte vital extracorpóreo para el síndrome de dificultad respiratoria aguda inducido por pancreatitis aguda es beneficioso. Se ha demostrado que la titulación de la noradrenalina a un PAM tan bajo como 65 $\mathrm{mmHg}$ conserva la perfusión tisular. La noradrenalina es el vasopresor recomendado en tales situaciones. (Dellinger, Carlet, \& Masur, 2004).

El tratamiento del síndrome de dificultad respiratoria del adulto se realiza según las directrices estándar. Recientemente, "se ha demostrado que el soporte vital extracorpóreo para el síndrome de dificultad respiratoria aguda inducido por pancreatitis aguda es beneficioso" (Banks, y otros, 2013). Al igual que la titulación de la noradrenalina a un PAM tan bajo como $65 \mathrm{mmHg}$ se ha demostrado que conserva la perfusión tisular. La noradrenalina es el vasopresor recomendado en tales situaciones. El tratamiento del síndrome de dificultad respiratoria del adulto se realiza según las directrices estándar.

\section{Papel de la hemodiafiltración continua o la hemofiltración venovenosa continua}

Dado que se cree que la hipercitocinemia es fundamental en la fisiopatología de la PA grave, se ha investigado el papel de la hemodiafiltración continua (CHDF) para la eli- 
minación de citocinas proinflamatorias. "El CHDF con una membrana de polimetilmetacrilato (PMMA) elimina varias citocinas del torrente sanguíneo y se usa ampliamente en Japón para la terapia de purificación de sangre en pacientes con afecciones mórbidas y se cree que previene la insuficiencia orgánica" (Banks, y otros, 2013).

\section{Objetivos de resucitación}

La reanimación con líquidos debe controlarse mediante la evaluación periódica de las funciones cardiovasculares, renales y pulmonares, así como de los desequilibrios electrolíticos. A menudo Trepte, Bachmann, \& Stork, (2013) "ha recomendado una disminución del hematocrito y BUN como marcador de corrección de la hemoconcentración". El hematocrito se ha utilizado durante más de 50 años para guiar la reposición de líquidos en pacientes críticamente enfermos; en PA, se ha identificado como un marcador que se correlaciona con el desarrollo de necrosis pancreática.

La falta de disminución del hematocrito se ha correlacionado con un aumento de la necrosis y un mal pronóstico. De manera similar, el BUN elevado se ha utilizado como marcador de enfermedad grave, y se ha informado que el BUN no ha disminuido en pacientes con necrosis aumentada. (Trepte, Bachmann, \& Stork, 2013).

El objetivo de la monitorización es la predicción precisa de la respuesta a los fluidos antes de que se produzca la expansión de volumen. "Los parámetros estáticos clásicos para la monitorización son la presión venosa central (PVC), la presión de oclusión de la arteria pulmonar (PAOP) y la PAM. Sin embargo, estos pueden ser falaces en pacientes que reciben ventilación mecánica y en aquellos con HAl" (Trepte, Bachmann, \& Stork, 2013). Los parámetros dinámicos que miden la respuesta cardíaca a los cambios en la precarga, como la variación del volumen sistólico y la variación de la presión del pulso (VPP), pueden utilizarse en pacientes con ventilación mecánica, pero requieren precaución en aquellos con $\mathrm{HAl}$.

Aunque la PVC se utiliza a menudo en unidades de cuidados intensivos para monitorizar la reanimación con líquidos, no es un punto final ideal en pacientes con PA porque existe una desconexión entre el espacio intracelular y el compartimento intravascular. Una CVP elevada en pacientes con PA grave puede indicar repleción intravascular cuando el compartimento intracelular en realidad está subresucitado.

Debido a la influencia combinada de la ventilación mecánica, IAH, derrame pleural y edema mediastínico, los parámetros basados en la presión pueden no reflejar con precisión la adecuación de la reposición de líquidos en AP. Por tanto, se han sugerido medidas hemodinámicas más nuevas, como el índice de volumen sanguíneo intratorácico, el índice de volumen tele diastólico global y el índice de agua pulmonar extravascular. Sin embargo, es necesario tener más datos y el hecho de que estas medidas sean invasivas hace que sea menos probable que se utilicen de forma rutinaria.

\section{Capacidad de respuesta a la fluidoterapia}

La capacidad de respuesta a la reanimación con líquidos se puede evaluar midiendo el hematocrito, BUN, producción de orina o índice de volumen intratorácico, entre otros parámetros. Como se destacó anteriormente, Wu \& Conwell, (2010)

Han utilizado BUN para clasificar a los pacientes como sensibles o refractarios a la reanimación con líquidos. Se ha sugerido una simple maniobra de elevación de la pierna para determinar la capacidad de respuesta a los líquidos en pacientes con respiración espontánea. Un aumento $\geq 10 \%$ en el gasto cardíaco durante los primeros 60-90 s de elevación de la pierna puede predecir la respuesta a la reposición de lí-

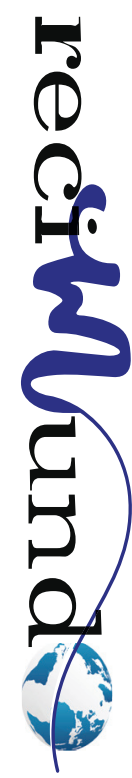


quidos. (p. 25)

\section{Seguimiento de la hipertensión intraab- dominal}

La PA grave puede complicarse con la HAI, que puede empeorar con la reanimación intensiva con líquidos.

En individuos sanos, la PIA varía de $0 \mathrm{mmHg}$ a $5 \mathrm{mmHg}$ y varía con el ciclo respiratorio. El IAH es un aumento sostenido de $\mid A P>12$ $\mathrm{mmHg}$. El síndrome compartimental abdominal es un aumento sostenido de la PIA> $20 \mathrm{mmHg}$, con insuficiencia orgánica de nueva aparición con o sin presión de perfusión abdominal baja. (Gardner, Vege, Pearson, \& Chari, 2018)

La incidencia de HAl en pacientes con PA grave es aproximadamente del 60 al $80 \%$. La HAl suele ser un fenómeno temprano, "en parte relacionado con los efectos del proceso inflamatorio que causa edema retroperitoneal, acumulación de líquido, ascitis e íleo y es en parte iatrogénico, como resultado de una reanimación agresiva con líquidos" (Mentula \& Leppäniemi, 2014). Se asocia con disfunción orgánica alterada, especialmente de los sistemas cardiovascular, respiratorio y renal.

Dado que contribuye a la falta de respuesta a los líquidos y conlleva una alta mortalidad quirúrgica, la $\mathrm{HAl}$ debe vigilarse en todos los pacientes con PA grave. La presencia de "presión intraabdominal> $16 \mathrm{mmHg}$ dificulta la interpretación correcta de los resultados de la elevación pasiva de la pierna, así como de otros parámetros de presión estática y dinámica" (Mentula \& Leppäniemi, 2014). La presión de perfusión abdominal puede servir como un buen marcador y punto final de reanimación en pacientes con HAl.

Se recomienda mantener la presión de perfusión abdominal por encima de 50-60 $\mathrm{mmHg}$ para facilitar la perfusión adecuada de los órganos abdominales. Hay algunas pruebas de que el uso de la reanimación con HES reduce el riesgo de $\mathrm{HAl}$ en la PA grave; sin embargo, el HES en realidad puede aumentar la mortalidad en pacientes críticamente enfermos. (Banks, y otros, 2013)

\section{Frecuencia de seguimiento}

Dado que la patogenia de la PA es un proceso dinámico, con complicaciones locales y sistémicas que agravan el manejo clínico, es imperativo controlar de cerca la reanimación con líquidos.

Después de un bolo inicial de $20 \mathrm{ml} / \mathrm{kg}$ o $1000 \mathrm{ml}$ durante una hora, seguido de una infusión controlada de líquidos durante las primeras $24 \mathrm{~h}$, los pacientes deben ser evaluados de cerca en una unidad de alta dependencia. Hemodinámicamente, los objetivos elegidos para monitorizar deben evaluarse en intervalos de 8 a 12 h. (Wu \& Conwell, 2010)

Los autores Wu \& Conwell, (2010) evaluaron a sus pacientes cada $8 \mathrm{~h}$ durante las primeras $24 \mathrm{~h}$. Aunque no existen directrices sobre este tema, parece lógico seguir esta práctica.

\section{Conclusión}

Durante el desarrollo de la investigación se observó que la reanimación con líquidos se ha convertido en una estrategia terapéutica clave en pacientes con pancreatitis aguda. Sin embargo, hay que reconocer que la reanimación con líquidos en la PA es un proceso complejo, por lo que es necesario tener en cuenta la dinámica de líquidos durante las diferentes etapas de la enfermedad.

Los conocimientos actuales sugieren que la reanimación con líquidos controlada (3,04,0 L / 24 h) debe iniciarse después de una infusión en bolo de $20 \mathrm{ml} / \mathrm{kg}(1000 \mathrm{ml}$ durante una hora). Entre los diferentes fluidos, el lactato de Ringers es el recomendado por la mayoría de las guías. Es necesario realizar la reanimación con líquidos con una 
estrategia dirigida por objetivos, con una producción de orina $>0,5 \mathrm{ml} / \mathrm{kg}$ y una disminución de BUN como objetivos simples.

Es obligatorio realizar ensayos controlados aleatorios para generar datos sobre los tres temas abordados (tasa, tipo y puntos finales de la reanimación con líquidos) antes de que se puedan enmarcar pautas definitivas.

\section{Bibliografía}

Banks, P., \& Freeman, L. (2016). Practice guidelines in acute pancreatitis. Am J Gastroenterol, 23792400 .

Banks, P., Bollen, T., Dervenis, C., Gooszen, H., Johnson, C., Sarr, M., . . . Vege, S. (2013). Classification of acute pancreatitis--2012: revision of the Atlanta classification and definitions by international consensus. Gut, 102-111.

Dellinger, R., Carlet, J., \& Masur, H. (2004). Surviving Sepsis Campaign guidelines for management of severe sepsis and septic shock. Crit Care Med, 858-873

Foitzik, T., Buhr, H., Messmer, K., \& Herfarth, C. (2003). Isovolemic hemodilution with dextran 60 as treatment of pancreatic ischemia in acute pancreatitis. Clinical practicability of an experimental concept. Ann Surg , 369-374

Gardner, T., Vege, S., Pearson, R., \& Chari, S. (2018). Fluid resuscitation in acute pancreatitis. Clin Gastroenterol Hepatol, 1070-1076.

Hack, C., \& Zeerleder, S. (2011). The endothelium in sepsis: source of and a target for inflammation. Crit Care Med, S21-S27.

Javier, R., \& Ángel, E. (2015). Coloides versus cristaloides en fluidoterapia guiada por objetivos, revisión sistemática y metaanálisis. Demasiado pronto o demasiado tarde para obtener conclusiones. Scielo.

Johannes, W., Sun, X., \& Tabak, Y. (2008). The early prediction of mortality in acute pancreatitis: a large population-based study. Gut, 1698-1703.

Lowenfels, A., Maisonneuve, P., \& Sullivan, T. (2009). The changing character of acute pancreatitis: epidemiology, etiology, and prognosis. Curr Gastroenterol Rep, 97-103.

Mao, E., Tang, Y., Li, L., \& Qin, S. (2017). Strategy of controlling fluid resuscitation for severe acute pancreatitis in acute phase. Zhonghua Wai Ke Zazhi,
1331-1334

Mayer, J., Rau, B., Gansauge, F., \& Beger, H. (2010). Inflammatory mediators in human acute pancreatitis: clinical and pathophysiological implications. Gut, 546-552 .

Mentula, P., \& Leppäniemi, A. (2014). Position paper: timely interventions in severe acute pancreatitis are crucial for survival. World J Emerg Surg, 15.

Sarr, M. (2013). Early fluid "resuscitation/therapy" in acute pancreatitis: which fluid? What rate? What parameters to gauge effectiveness? Ann Surg, 189-190.

Sterns, R., Riggs, J., \& Schochet, S. (2006). Osmotic demyelination syndrome following correction of hyponatremia. N Engl J Med.

Tenner, S. (2014). Initial management of acute pancreatitis: critical issues during the first 72 hours. Am J Gastroenterol , 2489-2494.

Trepte, C., Bachmann, K., \& Stork, J. (2013). The impact of early goal-directed fluid management on survival in an experimental model of severe acute pancreatitis. Intensive Care Med.

Wu, B., \& Conwell, D. (2010). Update in acute pancreatitis. Curr Gastroenterol Rep, 83-90 .

Zhao, G., Zhang, J., \& Wu, H. (2015). Effects of different resuscitation fluid on severe acute pancreatitis. World J Gastroenterol, 2044-2052. 


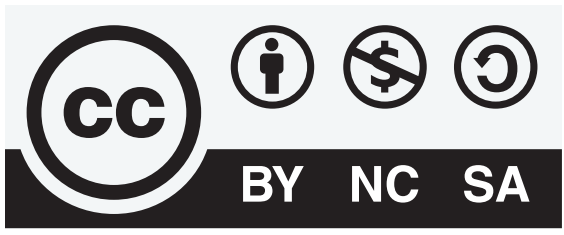

CREATIVE COMMONS RECONOCIMIENTO-NOCOMERCIAL-COMPARTIRIGUAL 4.0.

\section{CITAR ESTE ARTICULO:}

Díaz Castro, I., Jonathan Alberto, C., Erazo Haro, S., \& Matute Solis, M. (2020).

Manejo de hidratación en pancreatitis aguda. RECIMUNDO, 4(4), 46-58.

doi:10.26820/recimundo/4.(4).octubre.2020.46-58 